\title{
COLONIZATION AND ANTIBIOTIC RESISTANCE PROFILING OF METHICILLIN RESISTANT STAPHYLOCOCCUS AUREUS (MRSA) IN PATIENTS FROM TERTIARY CARE HOSPITAL, LAHORE, PAKISTAN
} Madiha Khan

Faculty of Life Sciences, University of Central Punjab, Lahore, Pakistan Wafa Komal

Faculty of Life Sciences, University of Central Punjab, Lahore, Pakistan Mushtaq A. Saleem

Faculty of Life Sciences, University of Central Punjab, Lahore, Pakistan Naveed Ahmed

Pakistan Kidney and Liver Institute \& Research Center, Lahore, Pakistan

Zara Rafaque

Faculty of Life Sciences, University of Central Punjab, Lahore, Pakistan

Saba Kabir

Faculty of Life Sciences, University of Central Punjab, Lahore, Pakistan

Department of Microbiology and Molecular Genetics, University of the Punjab, Lahore, Pakistan

\author{
Abdul Rehman* \\ Department of Microbiology and Molecular Genetics, University of the Punjab, Lahore, Pakistan \\ *Corresponding author \\ Department of Microbiology and Molecular Genetics, University of the Punjab, Quaid-e-Azam Campus, Lahore-54590, Pakistan. \\ Telephone: Telephone: 92429231249; Email: rehman.mmg@pu.edu.pk
}

\begin{abstract}
The methicillin resistant Staphylococcus aureus (MRSA) is affecting the economy worldwide contributing to the increased morbidity and treatment costs. The present cross sectional study was conducted to determine the association of MRSA with kidney and liver patients from Tertiary Care Hospital in Lahore, Pakistan and its antibacterial resistance patterns. The study was conducted for the period of one year; from April 2019 to April 2020. A total of 1984 different samples were obtained and examined for bacterial cultures using standard procedures. From total samples, $51.05 \%$ were collected from male and the rest $48.95 \%$ were collected from females including different age groups. Samples were inoculated on different culture media for isolation of $S$. aureus and susceptibility testing. A total of 253/1984 clinical samples exhibited $S$. aureus characteristics while 39 of 253 S. aureus (15.41\%) were identified as MRSA. MRSA strains were found highly resistant to Amikacin (AK), Cefoxitin (FOX), Clindamycin (DA), Fusidic acid (FD), Gentamicin (CN) and Tobramycin (TOB) while highest sensitivity was noted against Vancomycin (100\%) and Linezolid (100\%). The presence of MRSA and multidrug resistance pose serious therapeutic burden to critically ill patients. A systematic and concerted effort is essential to identify high-risk
\end{abstract}


patients rapidly and to reduce the burden of antimicrobial resistance.

Keywords: methicillin resistant Staphylococcus aureus; multidrug resistance; colonization; prevalence.

\section{Introduction}

Staphylococcus aureus (S. aureus) is a part of normal microflora of the nose, skin and respiratory tract in humans. About $20-30 \%$ of the total human population is a carrier of S. aureus where the nasal cavity remains the most common site for colonization (Banning. 2005; Sakr et al., 2018). S. aureus can cause several infections ranging from superficial skin infections to invasive life-threatening diseases such as pneumonia, bacteremia, endocarditis, meningitis, sepsis and toxic shock syndrome (Tong et al., 2015, Cadena et al., 2016). It has high ability to develop resistance to frequently used antibiotics. The use of antibiotics promotes resistance and influences co-selection processes of antibiotic resistant bacteria leading to transmission of resistance genes in the bacterial community (Bengtsson et al., 2018). S. aureus strains that are generally resistant to $\beta$-lactam antibiotics such as methicillin and oxacillin are referred to as MRSA. The resistance to $\beta$-lactams is mostly conferred by penicillin binding protein (PBP2a) encoded by mecA gene (Fuda et al., 2004). The MRSA strains are associated with higher mortality rates than methicillin-sensitive S. aureus (MSSA) (Hassoun et al., 2017).

The prevalence and resistance of $S$. aureus is associated with three types of infections i.e. hospital acquired (HA), healthcare-associated (HC) and community-acquired (CA) infection. Generally, HA-MRSA infections arise from persistent carriers those undergoing antibiotic therapy or from intermittent carriers. Both types of the carriers are at high risk of developing MRSA systemic infection such as bacteremia (Vigil et al., 2015). The healthcare-associated community onset infections are usually associated with comorbidities, such as chronic renal disease, diabetes and ulcers. MRSA can colonize Kidney and Liver transplant patients. The factors which increase the risk of colonization of organisms include antimicrobial use, use of drains and catheters, length of surgery and duration of stay at hospital (Oliveira et al., 2013). It has previously been reported that $10 \%$ to $25 \%$ of all liver transplant recipients are likely to acquire $S$. aureus infections. Moreover, the mortality rate may exceed $80 \%$ of all liver transplant recipients with MRSA pneumonia or MRSA intraabdominal infection (Singh et al., 2000).

Keeping in view the significance of MRSA in liver and kidney patients the current study was designed to determine the prevalence of MRSA and vancomycin resistance infection in kidney and liver settings at a Tertiary Care Hospital in Lahore, Pakistan.

\section{Methods}

\section{Collection of samples}

The samples were collected from liver and kidney patients from the Tertiary Care Hospital in Lahore. A total number of 1984 samples were obtained from these patients. The various samples included in study were Urine (704), Folly's Tip (101), cystoscopy urine (32), nephrostomy urine (54), pus (114), blood (654), wound swabs (89) and sputum (236). 


\section{Processing of samples}

After the collection of samples, all were transported to the laboratory of University of Central Punjab, Lahore for further processing. The isolation and identification of pathogenic bacteria was done using various culture media. The Cystine Electrolyte Deficient (CLED) agar was used for the processing of urine samples. Blood agar and MacConkey agar were used to inoculate samples other than urine. After inoculation, the samples were incubated for $18-24 \mathrm{~h}$ at $37^{\circ} \mathrm{C}$.

\section{S. aureus identification}

Biochemical profiling was done by various tests including catalase, coagulase and DNase test using Bergey's manual identification scheme (Bergey et al., 1939).

\section{Antibiotic susceptibility testing}

The Kirby Bauer disk diffusion method was used for antibiotic susceptibility testing. Various common antibiotics used for all sample types were Amikacin $(30 \mu \mathrm{g})$, Cefoxitin $(30 \mu \mathrm{g})$, Ciprofloxacin $(5 \mu \mathrm{g})$, Co-trimoxazole $(1.25 / 23.75 \mu \mathrm{g})$, Gentamicin $(10 \mu \mathrm{g})$, Linezolid $(30 \mu \mathrm{g})$, Erythromycin $(15 \mu \mathrm{g})$, Clindamycin $(2 \mu \mathrm{g})$, Penicillin (10units), Tetracycline (30 $\mu \mathrm{g})$, Teicoplanin $(30 \mu \mathrm{g})$, and Tobramycin $(10 \mu \mathrm{g})$.

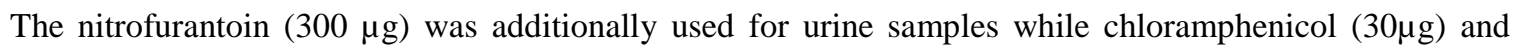
Fusidic acid $(10 \mu \mathrm{g})$ were used for samples other than urine. The results were interpreted in accordance with CLSI Guidelines (Clinical and Laboratory Standards Institute, 2015). All S. aureus isolates were classified into two groups according to presence of oxacillin resistance as MRSA and methicillin sensitive S. aureus (MSSA). The strain was considered MDR when it was found resistant to three or more of the tested antibiotics.

\section{E-Strip test}

The MRSA isolates were further tested for resistance against vancomycin (VRSA) by using Estrip (Biomeriux) determining minimum inhibitory concentration (MIC). The strains with MIC $\geq 16 \mu \mathrm{g} / \mathrm{ml}$ were considered as vancomycin resistant.

\section{Statistical analysis}

The percentage sensitivity data of antibiotic resistance was analyzed using SPSS version 20.0.

\section{Results}

\section{Demographic characteristics}

The highest positive isolates were obtained from females $17.40 \%$ (169/971). The male population is presented with almost half the number of $S$. aureus isolates than female population. The age group 40-49 showed strongest association with $S$. aureus positive isolates while this association was less common below 30 years of the age (Table 1$)$. 
Table 1: Demographic characteristics of S. aureus isolates.

\begin{tabular}{llll}
\hline & Variables & Total Samples & Positive for S. aureus \\
\cline { 2 - 4 } Gender & Male & 1013 & $84(8.29 \%)$ \\
\cline { 2 - 4 } & Female & 971 & $169(17.40 \%)$ \\
\cline { 2 - 4 } Age (Yrs) & $\mathbf{0 - 9}$ & 29 & $01(4.3 \%)$ \\
\cline { 2 - 4 } & $\mathbf{1 0 - 1 9}$ & 112 & $02(1.7 \%)$ \\
\cline { 2 - 4 } & $\mathbf{2 0 - 2 9}$ & 246 & $04(1.6 \%)$ \\
\cline { 2 - 4 } & $\mathbf{3 0 - 3 9}$ & 237 & $14(5.9)$ \\
\cline { 2 - 4 } & $\mathbf{4 0 - 4 9}$ & 556 & $106(19 \%)$ \\
\hline
\end{tabular}

\section{S. aureus isolation and identification}

A total of 253 out of 1984 samples were confirmed as S. aureus by macroscopic and microscopic characteristics followed by biochemical testing including coagulase, catalase and DNase tests. The maximum number of S. aureus isolates was obtained from cystoscopy urine comprising of (42.8\%), folly's tip (24\%), nephrostomy urine (22.2\%) followed by pus (18.4\%), wound swabs (15.7\%), urine (11.7\%), blood (11.6\%) and sputum (3.3\%) (Table 2).

Table 2: Prevalence of MRSA strains in different clinical samples.

\begin{tabular}{llll}
\hline \multicolumn{1}{c}{ Sample } & $\begin{array}{c}\text { Total samples } \\
(\mathbf{n = 1 9 8 4 )}\end{array}$ & S. aureus Positive $(\mathbf{n = 2 5 3 )}$ & MRSA (n=39) \\
\hline Urine & $704(35.5 \%)$ & $83(11.7 \%)$ & $14(16.86 \%)$ \\
\hline Folly's Tip & $101(5.1 \%)$ & $24(24.0 \%)$ & 0 \\
\hline Cystoscopy urine & $32(1.6 \%)$ & $15(42.8 \%)$ & $1(6.66 \%)$ \\
\hline Nephrostomy urine & $54(2.7 \%)$ & $12(22.2 \%)$ & $1(8.33 \%)$ \\
\hline Pus & $114(5.7 \%)$ & $21(18.4 \%)$ & $4(19.04 \%)$ \\
\hline Blood & $654(33.0 \%)$ & $76(11.6 \%)$ & $9(11.84 \%)$ \\
\hline Wound Swabs & $89(4.5 \%)$ & $14(15.7 \%)$ & $2(14.28 \%)$ \\
\hline Sputum & $236(11.9 \%)$ & $08(3.3 \%)$ & $8(100 \%)$ \\
\hline
\end{tabular}

\section{Antibiotic susceptibility testing}

All the biochemically confirmed $S$. aureus isolates were analyzed for MRSA by resistance to oxacillin disc. Out of 253 S. aureus strains, 39 (15.41\%) were resistant to oxacillin recognized as MRSA while the remaining 214 were identified as MSSA. The prevalence of MRSA strains of S. aureus isolated from different clinical samples is illustrated in table 2. All 8 strains isolated from sputum samples were MRSA while no MRSA strain was isolated from Folly's tip. Moreover, these MRSA strains were also tested for MDR using antibiotic discs. Figure 1 shows the representative plate of antibiotic susceptibility pattern to different antibiotics against Staphylococcus aureus. 


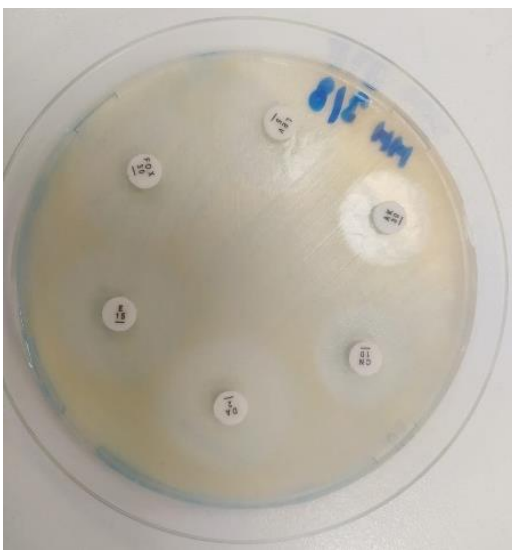

Figure 1: Staphylococcus aureus representative plate of antibiotic susceptibility pattern to different antibiotics.

Table 3 manifested the resistance pattern of MRSA and MSSA strains to various antibiotics. MRSA strains were found totally resistant to Amikacin (AK), Cefoxitin (FOX), Clindamycin (DA), Fusidic acid (FD), Gentamicin (CN) and Tobramycin (TOB). MSSA strains were observed with relatively high-level resistance to these drugs. A high antibiotic resistance of MRSA was also observed against Chloramphenicol (C) (36.5\%), Co-trimoxazole (SXT) (32\%), Erythromycin (E) (35\%) and Ciprofloxacin (CIP) (16\%) and Tetracycline (TE) (11.2\%). Two drugs Linezolid and Vancomycin were observed as highly sensitive (100\%) among our isolates and remains as the drug of choice for treating these patients (Table 3).

Table 3: Percentage of $S$. aureus resistance pattern to various antimicrobial agents.

\begin{tabular}{llll}
\hline \multirow{2}{*}{ Antibiotics } & Sensitive & \multicolumn{2}{c}{ Resistance } \\
\cline { 3 - 4 } & & $16(100)$ & MSSA (\%) \\
\hline Amikacin (AK) & 237 & $19(36.5)$ & $0(0)$ \\
\hline Chloramphenicol (C) * & 201 & $39(100)$ & $0(0)$ \\
\hline Cefoxitin (FOX) & 214 & $19(16)$ & $98(84)$ \\
\hline Ciprofloxacin (CIP) & 156 & $20(32)$ & $42(68)$ \\
\hline Co-trimoxazole (SXT) & 191 & $17(100)$ & $0(0)$ \\
\hline Clindamycin (DA) & 236 & $29(35)$ & $53(65)$ \\
\hline Erythromycin (E)* & 171 & $21(100)$ & 0 \\
\hline Fusidic acid (FD) $*$ & 232 & $24(100)$ & 0 \\
\hline Gentamicin (CN) & 229 & 0 & $178(100)$ \\
\hline Linezolid (LZD) ** & 39 & N/A & $111(88.8)$ \\
\hline Penicillin (P) & 36 & $14(11.2)$ & 0 \\
\hline Tetracycline (TE) & 128 & 0 & 0 \\
\hline Teicoplanin (TEC) & 253 & $16(100)$ & $145(100)$ \\
\hline Tobramycin (TOB) & 237 & N/A (0) & 0 \\
\hline Nitrofurantoin (F) & 69 & 0 & \\
\hline Vancomycin (VA) ** & 39 & & 0
\end{tabular}

*Not reported in urinary isolates **Reported in MRSA only

\section{Vancomycin resistance screening}

MRSA strains were tested for vancomycin resistance to detect the VRSA emergence among MRSA strains. No VRSA strain was isolated as all isolates were sensitive for vancomycin. 


\section{Discussion}

MRSA is involved in multiple infections starting from common infections like skin infections to life threatening infections such as meningitis, kidney failure, lung abscess and endocarditis. It is one of the most common infections encountered in health care settings and results in huge economic burden. Patients with chronic kidney obstruction and liver diseases are immunocompromised and the propensity of them acquiring HAI MRSA is even higher. Thus, it becomes very imperative to analyze the prevalence of MRSA in such patients to introduce early and appropriate antimicrobial therapy. The current study was designed to investigate the prevalence of methicillin resistance pattern in kidney and liver patients. Our results showed an overall $12.7 \%$ prevalence of $S$. aureus in different hospital samples, in which the highest percentage (42.8\%) was recovered from cystoscopy urine samples as compared to pus and blood samples (Table 1). This suggests that although $S$. aureus may not be the typical etiological agent involved in urinary tract infections, there are high chances of their presence in patients that are catheterized or go through cystoscopy procedures (Stokes et al., 2019).

The highest incidence of $S$ aureus among middle age (40-49 yr; 19\%) and aged people (>50: $15.6 \%)$ as compared to the children (0-12 yr; $1.5 \%)$ teenagers (13-19 yr; $1.7 \%)$ and young adults (20-39 yr; 5.9 ) indicate the increased vulnerability of $S$. aureus infection with age. The elderly people develop more chronic diseases and bacteremia as a result of comorbid conditions such as smoking, organ transplant, dysfunctional kidneys and livers, catheterization or prior hospitalization. Our study is in good agreement with other studies which have reported higher prevalence of $35.7 \%$ S. aureus mediated infections among elderly ( $>65 \mathrm{yr}$ ) as compared to the young adults. The higher percentage (17.4\%) of female patients infected with $S$. aureus as compared to male counterparts (8.29\%) is also in close alignment with this study, where they have reported higher percentage (45.3\%) of females infected with $S$. aureus as compared to males (39.7\%) (Kang et al., 2011).

The present study reports highest resistance against penicillin, (70\%), nitrofurantoin (57\%), tetracycline (49.4\%), ciprofloxacin (46\%), co-trimoxazole (42\%), and erythromycin (32.4\%). Our results show an increased resistance as compared to another study reported from Pakistan in 2014 (Khan, Rasheed and Zahra. 2014). The study by Khan et al. (2014) reported $12.2 \%$ and $28.9 \%$ resistance against tetracycline and erythromycin respectively, which is plausibly lower than our study, indicating high resistance among $S$. aureus isolates with passing time. MRSA is of great concern in kidney and liver patients because of their immunocompromised state. MRSA in such settings often lead to life threatening infections.

Our study reports, over-all $15.4 \%$ MRSA, which is in agreement with the surveillance study conducted in year 2018 and reports $26.3 \%-100 \%$ MRSA in liver recipient patients. The study also reports $1-23.5 \%$ in liver cirrhosis patients which is in close association with our results (Liu, Zhang and Wan. 2018). Unfortunately, there are no studies in Pakistan from liver and kidney settings to support our data. However, another study reports 33.9\% MRSA isolated from pus, blood, sputum, tracheal aspirate and urine (Ahmed et al., 2014). In another study pus and wound samples collected from patients with diagnosed 
malignancies had 38/68 S. aureus isolates that were recognized as MRSA (Duwadi et al., 2020). When tested against different drugs, MRSA were found significantly more resistant against amikacin (100\%), cefoxitin (100\%0, clindamycin (100\%), gentamicin (100\%), fusidic acid (100\%) and tobramycin (100\%) as compared to other drugs such as chloramphenicol (36.5\%), ciprofloxacin (16\%) and tetracycline (11.2\%) among others.

In this study the highest sensitivity of MRSA was noted against vancomycin (100\%) and linezolid (100\%) suggesting that these two drugs seem the choice of drugs under prevailing MRSA conditions. However, efforts need to be combated against the possible arise of VRSA which has been reported in other studies from Pakistan.

\section{Conclusion}

In conclusion, this study suggested the frequent colonization of MRSA in liver and kidney patients. These critically ill individuals are at high risk of developing severe infection with the resistant strains. Only limited data is available in Pakistan for the association of MRSA with liver and kidney patients. Therefore, further studies are required for effective monitoring of these patients to combat MRSA emergence and to control its spread and transmission in the community.

\section{Competing interests}

The authors declare that they have no competing interests.

\section{Authors' contributions}

MK, WK, and ZR performed experiments. MAS and NA analyzed the data. MK and SK wrote the manuscript. AR helped in manuscript editing. All authors approved the final manuscript.

\section{References}

Ahmed, A., Hussain, S., Ijaz, T. and Hashemy, I. 2014. Susceptibility of methicillin-resistant Staphylococcus aureus and Enterococci to teicoplanin in Pakistan: The MRSET study. Journal of Pakistan Medical Association, 64: 256-259.

Banning, M. 2005. Transmission and epidemiology of MRSA: current perspectives. British Journal of Nursing, 14: 548-554.

Bengtsson-Palm, J., Kristiansson, E. \& Larsson, D. J. 2018. Environmental factors influencing the development and spread of antibiotic resistance. FEMS Microbiology Reviews, 42: 68-80.

Bergey, D. H., Breed, R. S., Murray, E. G. D. \& Hitchens, A. P. 1939. Manual of determinative bacteriology. Manual of determinative bacteriology, Fifth Edn.

Cadena, J., Thinwa, J., Walter, E. A. \& Frei, C. R. 2016. Risk factors for the development of active methicillin-resistant Staphylococcus aureus (MRSA) infection in patients colonized with MRSA 
at hospital admission. Americam Journal of Infection Control, 44: 1617-1621.

Clinical and Laboratory Standards Institute (CLSI). 2015. Performance Standards for Antimicrobial Susceptibility Testing; Twenty-Fifth Informational Supplement M100-S25. 35.

Duwadi, K., Khadka, S., Adhikari, S., Sapkota, S. and Shrestha, P. 2020. Bacterial etiology of wound exudates in tertiary care cancer patients and antibiogram of the isolates. Infectious Diseases Research and Treatment; 13:1178633720952077.

Fuda, C., Suvorov, M., Vakulenko, S. B. \& Mobashery, S. 2004. The basis for resistance to $\beta$-lactam antibiotics by penicillin-binding protein 2a of methicillin-resistant Staphylococcus aureus. Journal of Biological Chemistry, 279: 40802-40806.

Hassoun, A., Linden, P. K. \& Friedman, B. 2017. Incidence, prevalence, and management of MRSA bacteremia across patient populations - a review of recent developments in MRSA management and treatment. Journal of Critical Care, 21: 211.

Kang, C-I., Song, J-H., Ko, K. S., Chung, D. R. \& Peck, K. R. 2011. Clinical features and outcome of Staphylococcus aureus infection in elderly versus younger adult patients. International Journal of Infectious Diseases, 15: 58-62.

Khan, S., Rasheed, F. \& Zahra, R. 2014. Genetic polymorphism of agr locus and antibiotic resistance of Staphylococcus aureus at two hospitals in Pakistan. Pakistan Journal of Medical Sciences, 30: 172-176.

Liu, T., Zhang, Y. \& Wan, Q. 2018. Methicillin-resistant Staphylococcus aureus bacteremia among liver transplant recipients: epidemiology and associated risk factors for morbidity and mortality. Infection and Drug Resistance, 11: 647-658.

Oliveira-Cunha, M., Bowman, V., Di Benedetto, G., Mitu-Pretorian, M., Armstrong, S., Forgacs, B., Tavakoli, A., Augustine, T. \& Pararajasingam, R. 2013. Outcomes of methicillin-resistant Staphylococcus aureus infection after kidney and/or pancreas transplantation. Transplantation Proceedings, 45: 2207-2210.

Sakr, A., Bregeon, F., Mege, J-L., Rolain, J-M. \& Blin, O. 2018. Staphylococcus aureus nasal colonization: an update on mechanisms, epidemiology, risk factors, and subsequent infections. Frontiers in Microbiology. 9: 2419 .

Singh, N., Paterson, D. L., Chang, F. Y., Gayowski, T., Squier, C., Wagener, M. M. \& Marino, I. R. 2000. Methicillin-resistant Staphylococcus aureus: the other emerging resistant gram-positive coccus among liver transplant recipients. Clinical Infectious Diseases, 30: 322-327.

Stokes, W., Parkins, M. D., Parfitt, E. C., Ruiz, J. C., Mugford, G. \& Gregson, D. B. 2019. Incidence and outcomes of Staphylococcus aureus bacteriuria: a population-based study. Clinical Infectious Diseases, 69: 963-969.

Tong, S. Y., Davis, J. S., Eichenberger, E., Holland, T. L. \& Fowler, V. G. 2015. Staphylococcus aureus infections: epidemiology, pathophysiology, clinical manifestations, and management. Clinical Microbiology Reviews, 28: 603-661. 
The Journal of Microbiology and Molecular Genetics (JMMG)

Vol. 1, Issue 3, Article No.4, (2020) pp: 31-39

Vigil, D. I., Harden, W. D., Hines, A. E., Hosokawa, P. W., Henderson, W. G. \& Bessesen, M. T. 2015. Risk of MRSA infection in patients with intermittent versus persistent MRSA nares colonization. Infection Control and Hospital Epidemiology, 36: 1292-1297. 